\title{
Multicommodity Lifetime Routing for Wireless Sensor Networks with Multiple Sinks
}

\author{
Vahid Shah-Mansouri, A. Hamed Mohsenian Rad, Vincent W.S. Wong \\ Department of Electrical and Computer Engineering \\ The University of British Columbia, Vancouver, Canada \\ e-mail: \{vahids, hamed, vincentw\}@ece.ubc.ca
}

\begin{abstract}
Wireless sensor networks (WSNs) have recently received increasing attention from research and development communities. In a WSN, the field information (e.g., temperature, humidity, airflow) is acquired via several battery-equipped wireless devices and is relayed towards a sink node. As the size of the WSNs increases, it becomes inefficient to gather all information in one sink. To tackle this problem, the number of sinks can be increased. The data information flow towards each of the sinks is called a commodity. In this paper, we formulate a lexicographically optimal commodity lifetime (LOCL) routing problem. A stepwise algorithm is proposed to obtain the optimal routing solution which can lead to lexicographical fairness among commodity lifetimes. Simulation results show that our proposed algorithm increases the normalized commodity lifetime compared to MLMS [1] and LMM [2] routing algorithms.
\end{abstract}

\section{INTRODUCTION}

Recent advances in low power integrated circuits have accelerated the development of various types of low cost wireless sensors, which are the building blocks for wireless sensor networks (WSNs). In WSNs, each node has the capability to sense the environment and relay the data towards the sink. Since most of the nodes are battery powered, one important design objective is to maximize the network lifetime (e.g., the time that the first sensor node runs out of its energy [3], [4]).

Various algorithms have been proposed to prolong the network lifetime in WSNs. In [5], Chang et al. formulated the maximum lifetime routing problem as a linear program (LP). In [6], Madan et al. proposed a distributed algorithm based on dual decomposition to solve the linear maximum lifetime problem. In [7], Nama et al. studied the utility-lifetime tradeoff by considering the source rates as variables in the maximum lifetime problem. In [2], Hou et al. proposed an iterative algorithm to obtain a lexicographic max-min fair routing solution. The fairness is achieved among the lifetime of different nodes. In [8], Dagher et al. proposed an iterative algorithm which leads to Pareto-optimal routing solutions for WSNs. In [9], we proposed the regularized maximum lifetime routing problem to jointly maximize the network lifetime and also minimize delay or power consumption in the network.

The algorithms in [3]-[8], [10] are proposed specifically for single sink WSNs. As the size of the network increases, it becomes inefficient (in terms of power consumption) and sometimes impossible (in terms of link capacities) to gather all the information in a single sink node. To tackle this problem, one can increase the number of sinks. A sample
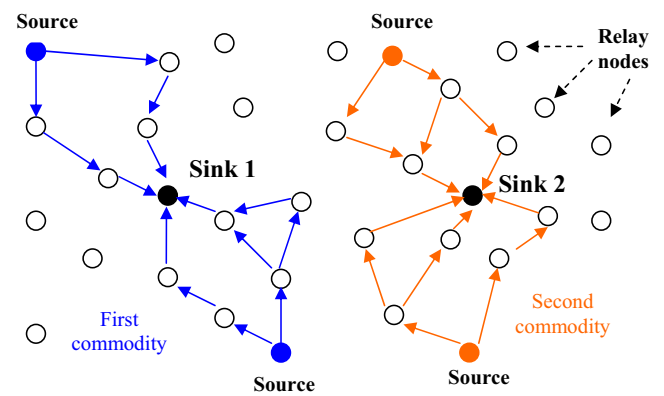

Fig. 1. A sample wireless sensor network with two sinks.

multiple sinks WSN with two sinks is illustrated in Fig. 1. We assume that each node sends data to the nearest sink. The data information flow towards each particular sink is called a commodity. In [11], Oyman et al. proposed a multiple sinks WSN architecture where the network is partitioned into clusters. All the sources in a cluster were assigned to send the data to the sink designated to that particular cluster. In [12], Thulasiraman et al. considered a multi-drain sensor network. Data from each source is logged into two distinct drains for data collection to be resilient to any single drain failure. In [13], the upper and lower bounds for the optimal solution of the multiple sink problem are obtained. It is shown that the bounds are tight for networks with a large number of nodes.

The main issue in obtaining the routing solution is fairness among different commodities. In this paper, we address the fairness issue and propose a lexicographically fair routing algorithm. The contributions of this paper are as follows:

- We consider the lifetime problem in a WSN with multiple sinks. We formulate this problem as a sequence of tractable optimization problems.

- We propose stepwise lexicographically optimal commodity lifetime (LOCL) algorithm to find the optimal solution. The obtained routing solution is called lexicographically optimal commodity lifetime routing (LOCLR).

- Using LOCL, the normalized commodity lifetime for networks with four sinks increases by $100 \%$ and $35 \%$ compared to the lifetime achieved by maximum lifetime multiple sinks (MLMS) [1] and lexicographical max-min fair (LMM) [2] algorithms, respectively.

The rest of the paper is organized as follows: In Section II, we formulate the problem and propose the stepwise algorithm to obtain the optimal routing solution. In Section III, we 
evaluate the performance of our proposed design scheme and compare it with two other methods. Conclusions are given in Section IV.

\section{Stepwise LeXicographically Optimal COMmodity Lifetime (LOCL) Algorithm}

\section{A. Problem Formulation}

Consider a WSN consisting of a set of sensor nodes $V$. Let $C$ denote the set of sinks collecting information from the network. Also let $S_{i}^{k}$ denote the data rate generated by source node $i$ and sent to sink $k \in C$. Let $N_{i}$ be the set of neighbors of node $i \in V$. Let $x_{i j}^{k}$ denote the data rate sending to sink $k$ from node $i$ to node $j \in N_{i}$. The aggregate data rate for the unidirectional logical link from node $i$ to $j \in N_{i}$ is denoted by $x_{i j}$ and is equal to $\sum_{k \in C} x_{i j}^{k}$. For notation simplicity, we stack up all $x_{i j}$ and denote them as vector $\mathbf{x}$. Let $p_{i j}$ denote the power consumed in node $i \in V$ for transmission of one bit information to node $j \in N_{i}$. The maximum data rate between nodes $i$ and $j$ is denoted as $R_{i j}$. Let $E_{i}$ represent the initial energy of node $i$. The lifetime of node $i$ is obtained as:

$$
T_{i}(\mathbf{x})=\frac{E_{i}}{\sum_{j \in N_{i}} p_{i j} \sum_{k \in C} x_{i j}^{k}} .
$$

The lifetime of commodity $k \in C$ under vector $\mathbf{x}$ becomes:

$$
T^{k}(\mathbf{x})=\min _{i \in V}\left\{T_{i}(\mathbf{x}) \mid i \in V \text { and } \sum_{j \in N_{i}} x_{i j}^{k}>0\right\} \text {. }
$$

Let $\mathbf{T}=\left(T^{1}, T^{2}, \cdots, T^{|C|}\right)$ denote the commodity lifetime vector in the network. It is called a lexicographically ordered commodity lifetime vector if $T^{1} \leq \cdots \leq T^{|C|}$. That is, all the elements in the vector are sorted in an ascending order. The commodity lifetime vector $\hat{\mathbf{T}}$ is lexicographically greater than vector $\tilde{\mathbf{T}}$ if and only if there exists $i$ such that $\hat{T}^{i}>\tilde{T}^{i}$, and for all $j<i$ we have $\hat{T}^{j}=\tilde{T}^{j}$. A vector is lexicographically optimal in a set if it is the lexicographically greatest vector in the set. Note that for any compact set of $\mathbb{R}^{m}$, there exists only one lexicographically optimal vector [14].

In our problem formulation, the objective is to determine the routing paths and flow rates which lead to the lexicographically greatest feasible lifetime vector. The LOCL algorithm is a stepwise algorithm. In the first step, the minimum commodity lifetime in the network is maximized. This step may have an infinite number of optimal routing solutions. In the second step, among the solutions from the first step, a solution is chosen which maximizes the second minimum commodity lifetime. The second step may also have an infinite number of optimal solutions. In general, in step $n$ of the LOCL algorithm, among the solutions from step $n-1$, the solution is chosen which maximizes the $n$th minimum commodity lifetime. The routing solution in the last step lexicographically maximizes the lifetime of all commodities.

Definition 1. A routing flow is LOCL Routing (LOCLR) if the vector of lifetimes of commodities under this routing flow is the lexicographically greatest feasible commodity lifetime vector.

\section{B. First Step}

We now present the first step of the LOCL algorithm and describe how it can be converted to a linear mixedinteger programming problem. In the first step, the minimum commodity lifetime is maximized. The problem is as follows:

$$
\begin{aligned}
\operatorname{maximize} & \min _{k \in C}\left\{T^{k}(\mathbf{x})\right\} \\
\text { subject to } & \sum_{j \in N_{i}}\left(x_{i j}^{k}-x_{j i}^{k}\right)=S_{i}^{k}, \quad \forall i \in V, \forall k \in C \\
& \sum_{k \in C} x_{i j}^{k} \leq R_{i j}, \quad \forall i \in V, \forall j \in N_{i} \\
& x_{i j}^{k} \geq 0, \quad \forall i \in V, \forall j \in N_{i}, \forall k \in C .
\end{aligned}
$$

The first set of constraints is the flow conservation for each commodity in all the nodes. The second set of constraints is the data rate limits on each link. To obtain a linear objective function, we introduce an auxiliary scalar variable $t$, which is a lower bound for the lifetime of minimum commodity. We replace the value of $T^{k}$ from equation (2) and reformulate problem (3) as follows:

$$
\begin{aligned}
& \text { maximize } t \\
& \text { subject to } t \leq T^{k}, \quad \forall k \in C \\
& T^{k}=\min _{i \in V}\left\{T_{i} \mid T_{i}=E_{i} /\left(\sum_{j \in N_{i}} p_{i j} \sum_{m \in C} x_{i j}^{m}\right)\right. \\
& \text { and } \left.\sum_{j \in N_{i}} x_{i j}^{k}>0\right\}, \quad \forall k \in C \\
& \sum_{j \in N_{i}}\left(x_{i j}^{k}-x_{j i}^{k}\right)=S_{i}^{k}, \quad \forall i \in V, \forall k \in C \\
& \sum_{k \in C} x_{i j}^{k} \leq R_{i j}, \quad \forall i \in V, \forall j \in N_{i} \\
& x_{i j}^{k} \geq 0, \quad \forall i \in V, \forall j \in N_{i}, \forall k \in C .
\end{aligned}
$$

The second constraint in problem (4) can be replaced by:

$$
T^{k} \leq E_{i} /\left(\sum_{j \in N_{i}} \sum_{m \in C} p_{i j} x_{i j}^{m}\right), \quad \text { if } \sum_{j \in N_{i}} x_{i j}^{k}>0, \forall i \in V \text {. }
$$

We replace $T^{k}$ and $t$ with their respective inverses: $q^{k}=$ $\frac{1}{T^{k}}$ and $q=\frac{1}{t}$. The objective function is changed from maximizing $t$ to minimizing $q$. The problem (4) can now be written as follows:

$$
\begin{aligned}
& \text { minimize } q \\
& \text { subject to } \\
& q^{k} \leq q, \quad \forall k \in C \\
& \sum_{j \in N_{i}} \sum_{m \in C} p_{i j} x_{i j}^{m} \leq E_{i} q^{k}, \text { if } \sum_{j \in N_{i}} x_{i j}^{k}>0, \forall i \in V, \forall k \in C \\
& \sum_{j \in N_{i}}\left(x_{i j}^{k}-x_{j i}^{k}\right)=S_{i}^{k}, \quad \forall i \in V, \forall k \in C \\
& \sum_{k \in C} x_{i j}^{k} \leq R_{i j}, \quad \forall i \in V, \forall j \in N_{i} \\
& x_{i j}^{k} \geq 0, \quad \forall i \in V, \forall j \in N_{i}, \forall k \in C .
\end{aligned}
$$


The second constraint in problem (5) is conditional. To obtain a closed form for this constraint, we introduce an auxiliary boolean variable $b_{i}^{k}$ where $b_{i}^{k}$ is equal to 1 when node $i$ carries information of commodity $k$. We can map the new boolean variable $b_{i}^{k}$ to rate variables as follows:

$$
\frac{\sum_{j \in N_{i}} x_{i j}^{k}}{\sum_{j \in N_{i}} R_{i j}} \leq b_{i}^{k}, \quad \forall i \in V, \quad \forall k \in C .
$$

The conditional constraint in (5) can now be written as:

$$
b_{i}^{k} \sum_{j \in N_{i}} \sum_{m \in C} p_{i j} x_{i j}^{m} \leq b_{i}^{k} E_{i} q^{k}, \quad \forall i \in V, \quad \forall k \in C .
$$

This constraint is still nonlinear. We use a linearization technique and convert it to a set of linear constraints. Details of the linearization technique can be found in [15]. We first define two auxiliary variables as follows:

$$
\begin{aligned}
& \nu_{i}^{k}=b_{i}^{k} \sum_{j \in N_{i}} \sum_{m \in C} p_{i j} x_{i j}^{m}, \quad \forall i \in V, \quad \forall k \in C \\
& \gamma_{i}^{k}=b_{i}^{k} E_{i} q^{k}, \quad \forall i \in V, \quad \forall k \in C .
\end{aligned}
$$

Based on these two new variables, constraint (7) for node $i$ and commodity $k$ would be $\nu_{i}^{k} \leq E_{i} \gamma_{i}^{k}$. A set of constraints is added for each new variable for commodity $k$ and node $i$ :

$$
\begin{gathered}
0 \leq \nu_{i}^{k} \leq \sum_{j \in N_{i}} \sum_{m \in C} p_{i j} x_{i j}^{m}, \\
\sum_{j \in N_{i}} p_{i j} \sum_{m \in C} x_{i j}^{m}-\left(1-b_{i}^{k}\right) P_{i}^{\text {max }} \leq \nu_{i}^{k}, \\
\nu_{i}^{k} \leq b_{i}^{k} P_{i}^{\max }, \\
0 \leq \gamma_{i}^{k} \leq q^{k}, \\
q^{k}-q_{\max }^{k}\left(1-b_{i}^{k}\right) \leq \gamma_{i}^{k} \leq q_{\max }^{k} b_{i}^{k},
\end{gathered}
$$

where $P_{i}^{\max }=\sum_{j \in N_{i}} p_{i j} R_{i j}$ and $q_{\max }^{k}$ is a loose upper bound for $q^{k}$. Problem (5) is formulated with new variables:

minimize $q$

subject to

$q^{k} \leq q$,

$\forall k \in C$

$\sum_{j \in N_{i}}\left(x_{i j}^{k}-x_{j i}^{k}\right)=S_{i}^{k}, \quad \forall i \in V, \forall k \in C$

$\frac{\sum_{j \in N_{i}} x_{i j}^{k}}{\sum_{j \in N_{i}} R_{i j}} \leq b_{i}^{k}$,

$\forall i \in V, \forall k \in C$

$\nu_{i}^{k} \leq E_{i} \gamma_{i}^{k}$,

$\forall i \in V, \forall k \in C$

$0 \leq \nu_{i}^{k} \leq \sum_{j \in N_{i}} \sum_{m \in C} p_{i j} x_{i j}^{m}, \forall i \in V, \forall k \in C$

$\sum_{j \in N_{i}} \sum_{m \in C} p_{i j} x_{i j}^{m}-\left(1-b_{i}^{k}\right) P_{i}^{\max } \leq \nu_{i}^{k}, \forall i \in V, \forall k \in C$

$\sum_{k \in C} x_{i j}^{k} \leq R_{i j}, \quad \forall i \in V, \forall j \in N_{i}$

$\nu_{i}^{k} \leq b_{i}^{k} P_{i}^{\max }, \quad \forall i \in V, \forall k \in C$

$0 \leq \gamma_{i}^{k} \leq q^{k}, \quad \forall i \in V, \forall k \in C$

$q^{k}-q_{\text {max }}^{k}\left(1-b_{i}^{k}\right) \leq \gamma_{i}^{k} \leq q_{\text {max }}^{k} b_{i}^{k}, \quad \forall i \in V, \forall k \in C$

$\sum_{k \in C} x_{i j}^{k} \leq R_{i j}, \quad \forall i \in V, \forall j \in N_{i}$

$x_{i j}^{k} \geq 0, b_{i}^{k} \in\{0,1\} \quad \forall i \in V, \forall j \in N_{i}, \forall k \in C$.
Because of the linearity of the objective function in problem (10), it is possible to have an infinite number of optimal commodity lifetime solutions. To obtain a unique set of commodities with minimum lifetime in this step and the subsequent steps, we use the regularization method. Details of the regularization technique can be found in [16]. The regularization term, $\delta \sum_{k \in C}\left(q^{k}\right)^{2}$, is the Euclidean norm of the commodity lifetime vector, where $\delta$ is the regularization coefficient. This regularization term only ensures that the optimal commodity lifetime vector is unique. It is still possible to have an infinite number of optimal routing paths.

\section{Subsequent Steps}

The first step in the LOCL algorithm is a linear mixedinteger program (MIP). The feasible set in the second step is the optimal solution set obtained from the first step. Similarly, the feasible set in the third step is the optimal solution obtained from the second step, and so on. We call the mixed integer programming problem in step $n$ as MIP- $n$.

Assume that the minimum lifetime in the first step (problem (10)) is $T^{1 \star}$ and the optimal value (inverse of minimum commodity lifetime) is $q^{1 \star}$. Let $\mathcal{P}_{1}$ be the set of commodities that the first constraint is active in problem (10). In the second step, the minimum lifetime among all the commodities except the members of $\mathcal{P}_{1}$ (i.e., $C \backslash \mathcal{P}_{1}$ ) is maximized subject to the condition that the lifetime of the commodities in $\mathcal{P}_{1}$ is also being maximized. The problem in the second step is similar to problem (3) while the objective is modified to be as follows:

$$
\operatorname{maximize} \min _{k \in C \backslash \mathcal{P}_{1}} T^{k}(\mathbf{x}) \text {. }
$$

Also, there is a constraint on the maximization of minimum lifetime. The constraint is:

$$
T^{l} \geq T^{1 \star}, \quad \forall l \in \mathcal{P}_{1} .
$$

The problem in the $n$th step can be formulated with similar changes in the objective function and by including the additional constraints. Let $T^{h \star}$ denote the maximum achievable value for the $h$ th minimum commodity lifetime (obtained from the $h$ th step). Let $\mathcal{P}_{h}$ denote the set of commodities that their lifetimes are equal to $T^{h \star}$ in the $h$ th step. We have $\mathcal{P}_{h}=\left\{k \mid T^{k}=T^{h \star}\right.$ and $\left.k \in C \backslash \bigcup_{l=1}^{h-1} \mathcal{P}_{l}\right\}$. The problem in the $n$th step is as follows:

$$
\begin{array}{rlr}
\operatorname{maximize} & \min _{k \in C \backslash \bigcup_{h-1}^{n-1} \mathcal{P}_{h}}\left\{T^{k}(\mathbf{x})\right\} & \\
\text { subject to } & T^{l} \geq T^{h \star}, \quad \forall l \in \mathcal{P}_{h}, h=1, \ldots, n-1 \\
& \sum_{j \in N_{i}}\left(x_{i j}^{k}-x_{j i}^{k}\right)=S_{i}^{k}, \quad \forall i \in V, \forall k \in C \\
& \sum_{k \in C} x_{i j}^{k} \leq R_{i j}, & \forall i \in V, \forall j \in N_{i} \\
& x_{i j}^{k} \geq 0, \forall i \in V, \forall j \in N_{i}, \forall k \in C .
\end{array}
$$

By letting $q^{h \star}=1 / T^{h \star}$, the same series of changes applied to problem (3) can be applied to problem (11). The mixed integer 
programming problem in step $n$ (i.e., MIP- $n$ ) is as follows:

$$
\begin{aligned}
& \text { minimize } q \\
& \text { subject to } \\
& q^{k} \leq q \\
& q^{l} \leq q^{h \star}, \\
& \sum_{j \in N_{i}}\left(x_{i j}^{k}-x_{j i}^{k}\right)=S_{i}^{k}, \quad \forall i \in V, \forall k \in C \\
& \frac{\sum_{j \in N_{i}} x_{i j}^{k}}{\sum_{j \in N_{i}} R_{i j}} \leq b_{i}^{k}, \\
& \nu_{i}^{k} \leq E_{i} \gamma_{i}^{k} \text {, } \\
& \forall i \in V, \forall k \in C \\
& 0 \leq \nu_{i}^{k} \leq \sum_{j \in N_{i}} \sum_{m \in C} p_{i j} x_{i j}^{m}, \forall i \in V, \forall k \in C \\
& \sum_{j \in N_{i}} \sum_{m \in C} p_{i j} x_{i j}^{m}-\left(1-b_{i}^{k}\right) P_{i}^{\max } \leq \nu_{i}^{k}, \forall i \in V, \forall k \in C \\
& \nu_{i}^{k} \leq b_{i}^{k} P_{i}^{\max }, \quad \forall i \in V, \forall k \in C \\
& 0 \leq \gamma_{i}^{k} \leq q^{k}, \quad \forall i \in V, \forall k \in C \\
& q^{k}-q_{\text {max }}^{k}\left(1-b_{i}^{k}\right) \leq \gamma_{i}^{k} \leq q_{\max }^{k} b_{i}^{k}, \quad \forall i \in V, \forall k \in C \\
& \sum_{k \in C} x_{i j}^{k} \leq R_{i j}, \quad \forall i \in V, \forall j \in N_{i} \\
& x_{i j}^{k} \geq 0, b_{i}^{k} \in\{0,1\} \quad \forall i \in V, \forall j \in N_{i}, \forall k \in C .
\end{aligned}
$$

\section{Stepwise Algorithm}

Algorithm 1 shows the stepwise algorithm which can be used to determine the LOCLR solution.

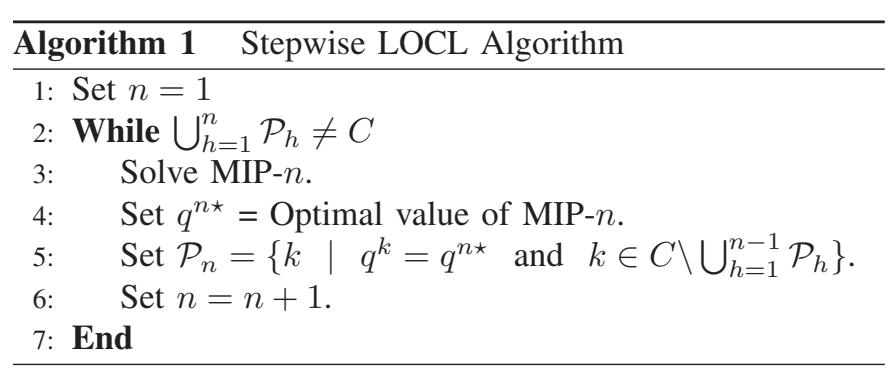

The MIP-1 is problem (10). The MIP- $n$ (for $n>1$ ) is problem (12). The number of steps in the stepwise LOCL algorithm is less than or equal to the number of commodities.

\section{Performance Evaluation}

In this section, we present the results of the proposed LOCL algorithm. We assume a deterministic path loss model. The power consumed for transmission of one bit from node $i$ to node $j$ (i.e., $p_{i j}$ ) is $\eta_{1}+\eta_{2} d_{i j}^{4}$, where $d$ is the physical distance. We choose $\eta_{1}=1$ and $\eta_{2}=0.1$. The regularization coefficient is set to $\delta=10^{-5}$. Each source generates $1 \mathrm{kbps}$ of information. The initial energy of each source node $E_{S}$ is 300 $\mathrm{J}$ while the initial energy of each intermediate node $i$ (i.e., $E_{i}$ ) is $100 \mathrm{~J}$. Nodes use time division multiple access (TDMA) to access the channel. The maximum link rate $R_{i j}$ is $250 \mathrm{kbps}$ for all logical links. In the stepwise LOCL algorithm, we solve the corresponding mixed integer programming problems by using the MOSEK [17] optimization toolbox.

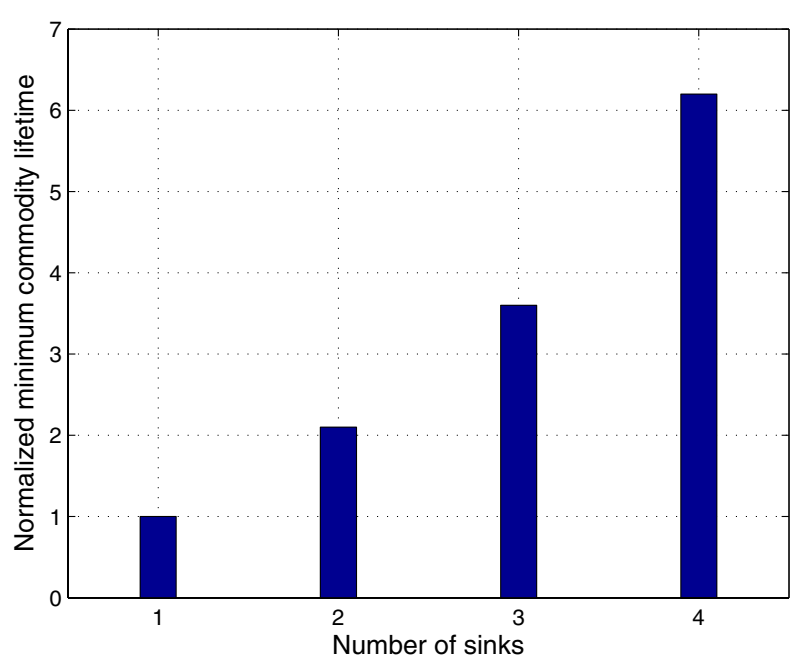

Fig. 2. Minimum commodity lifetime for network with different number of sinks.

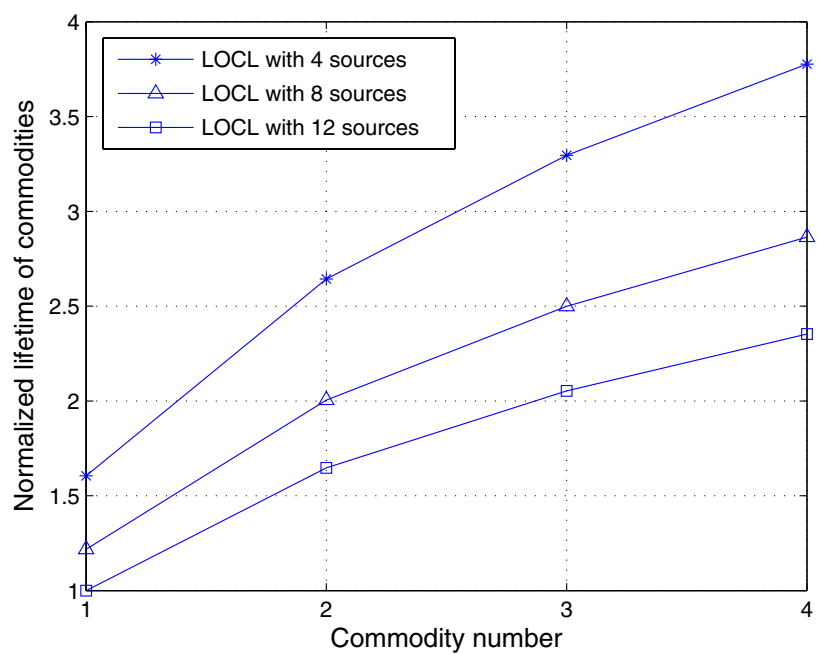

Fig. 3. Lifetime of commodities for LOCL algorithm with different number of sources.

In the first experiment, we show the performance of employing multiple sinks. There are 30 sensor nodes randomly deployed in a $50 \mathrm{~m} \times 50 \mathrm{~m}$ square field. The transmission range of each node is $10 \mathrm{~m}$. There exist four sinks located at the four corners of the field. Eight nodes are randomly chosen as sources. Each source in each step sends data to its (physically) closest sink. Fig. 2 shows the normalized minimum commodity lifetime in the network with different number of sinks. Results are averaged over 100 simulation runs. The values are normalized with respect to the lifetime of the network with only one sink. Simulation results depict that the lifetime increases almost linearly as the number of sinks is increased. It can be seen that the minimum commodity lifetime in the network with four sinks is almost 500\% higher compared to the network with only one sink.

Next, we investigate the performance of the LOCL algo- 


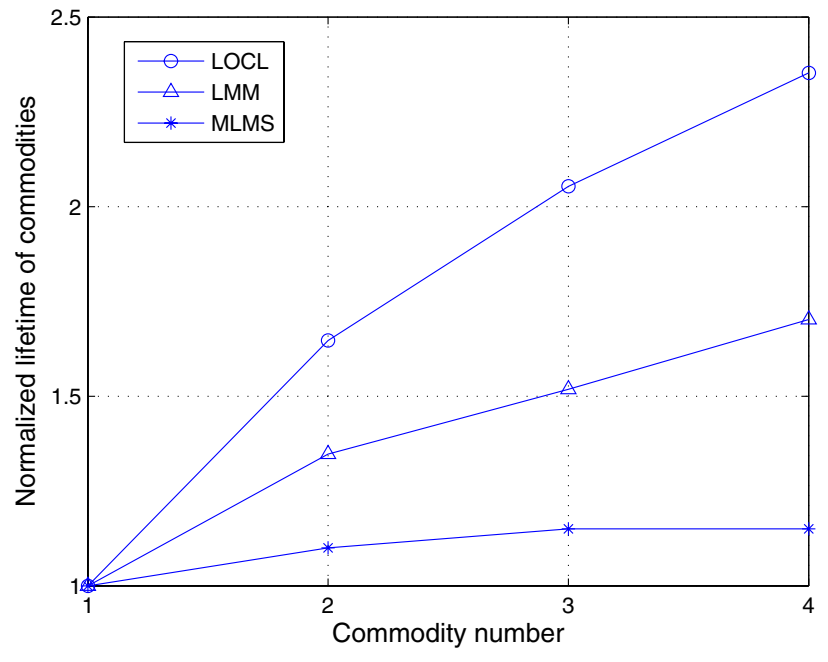

Fig. 4. Lifetime of commodities for LOCL, LMM, and MLMS algorithms.

rithm with different number of sources. The other simulation settings are the same as before. Keeping the number of sinks as four, we perform simulations when the number of sources varies from 4 to 12 . Fig. 3 shows the average normalized commodity lifetime for different commodities. When the number of sources increases, the lifetime of commodities decreases.

To compare the proposed algorithm with the existing algorithms in the literature, we implemented the algorithms proposed in [1] and [2]. In [1], the lifetime maximization problem is modeled as a concurrent multi-commodity flow optimization problem. The problem can be extended to consider the multisink case. We modify this algorithm and assume that each source sends data to the closest sink. We call the modified algorithm as the Maximum Lifetime routing problem for network with Multiple Sinks (MLMS). Lexicographical maxmin fair (LMM) algorithm is proposed in [2]. This algorithm determines a schedule for the routing flows. We extend this algorithm for WSNs with multiple sinks. We compare the results of LOCL with the first routing flow of LMM method.

There are four sinks and eight sources in the network. Fig. 4 compares the lifetime of commodities for different algorithms. In this figure, for the minimum commodity lifetime, all the algorithms have the same lifetime. The lifetime of commodities in MLMS algorithm are almost equal because this algorithm only maximizes the minimum lifetime in network. For LMM algorithm, the first routing flow from the schedule is compared. Results show the better performance of LOCL compared to these two algorithms. Comparing the results when the number of commodities is 4 , we can see that our proposed LOCL algorithm can increase the normalized lifetime of the forth commodity by $100 \%$ and $35 \%$ compared to algorithms MLMS and LMM, respectively.

\section{CONCLUSiONS}

Multiple sinks can be employed in a WSN to increase the lifetime of the network. In this paper, we formulated LOCL algorithm to fairly share the network resources among various commodities in the system using the concept of lexicographical fairness. This algorithm is a step-wise algorithm. In each step, a linear mixed-integer programming problem is being solved. Simulation results show that LOCL algorithm has better performance compared to some existing schemes.

There are several directions for future work. The algorithm can be extended to enable the source nodes to select the appropriate sink. Another direction is to determine the optimal location for the sinks while the network lifetime is being maximized.

\section{ACKNOWLEDGEMENT}

This work is supported by the Natural Sciences and Engineering Research Council of Canada (NSERC).

\section{REFERENCES}

[1] Y. Xue, Y. Cui, and K. Nahrstedt, "Maximizing lifetime for data aggregation in wireless sensor networks," ACM/Kluwer Mobile Networks and Applications (MONET), vol. 10, pp. 853-864, Dec. 2005.

[2] Y. Hou, Y. Shi, and H. D. Sherali, "On node lifetime problem for energyconstrained wireless sensor networks," ACM/Kluwer Mobile Networks and Applications (MONET), vol. 10, pp. 875-878, Dec. 2005.

[3] J. Chang and L. Tassiulas, "Routing for maximum system lifetime in wireless ad-hoc networks," in Proc. of Allerton Conference Communication, Control, and Computing, Urbana, Illinois, Sept. 1999.

[4] H. Zhang and J. C. Hou, "On deriving the upper bound of $\alpha$-lifetime for large sensor networks," in Proc. of ACM MobiHoc, Roppongi Hills, Japan, May 2004.

[5] J. Chang and L. Tassiulas, "Maximum lifetime routing in wireless sensor networks," IEEE/ACM Trans. on Networking, vol. 12, pp. 609-619, Aug. 2004

[6] R. Madan and S. Lall, "Distributed algorithms for maximum lifetime routing in wireless sensor networks," IEEE Trans. on Wireless Cотmunications, vol. 5, pp. 2185-2193, Aug. 2006.

[7] H. Nama, M. Chiang, and N. Mandayam, "Optimal utility-lifetime tradeoff in self-regulating wireless sensor networks a distributed approach," in Proc. of 40th Annual Conference on Information Sciences and Systems (CISS), Princeton, NJ, March 2006.

[8] J. C. Dagher, M. W. Marcellin, and M. A. Neifeld, "A theory for maximizing the lifetime of sensor networks," IEEE Trans. on Communications, vol. 55, pp. 323-332, Feb. 2007.

[9] V. Shah-Mansouri and V. W. S. Wong, "Distributed maximum lifetime routing in wireless sensor networks based on regularization," in Proc. of IEEE Globecom, Washington, DC, Nov. 2007.

[10] J. Zhu, S. Chen, B. Bensaou, and K. Hung, "Tradeoff between lifetime and rate allocation in wireless sensor networks: A cross layer approach," in Proc. of IEEE INFOCOM, Anchorage, Alaska, May 2007.

[11] E. I. Oyman and C. Ersoy, "Multiple sink network design problem in large scale wireless sensor networks," in Proc. of IEEE ICC, Paris, France, June 2004.

[12] P. Thulasiraman, S. Ramasubramanian, and M. Krunz, "Disjoint multipath routing to two distinct drains in a multi-drain sensor network," in Proc. of IEEE INFOCOM, Anchorage, Alaska, May 2007.

[13] V. Shah-Mansouri and V. W. S. Wong, "Bounds for lifetime maximization with multiple sinks in wireless sensor networks," in Proc. of IEEE Pacific Rim Conference on Communications, Computers and Signal Processing, Victoria, Canada, Aug. 2007.

[14] S. Sarkar and L. Tassiulas, "Fair bandwidth allocation of multicasting in networks with discrete feasible set," IEEE Transaction on Computers, vol. 53, pp. 785-797, July 2004.

[15] F. Glover, "Improved linear integer programming formulations of nonlinear integer problems," Management Science, vol. 22, pp. 455-460, Dec. 1975.

[16] O. Mangasarian and R. Meyer, "Nonlinear perturbation of linear programs," SIAM Journal on Optimization, vol. 17, pp. 745-752, 1977.

[17] http://www.mosek.com. 